\title{
Gravitationswellen - ein neues Fenster zum Universum
}

\author{
Peter A ufmuth und Albrecht Rüdiger
}

D ie von der A llgemeinen R elativitätstheorie vorhergesagten $G$ ravitationswellen sind bisher noch nicht direkt beobachtet worden. In Europa, Japan und den U SA hat man mit dem Bau von großen $L$ aserinterferometern begonnen, die dies ermöglichen sollen. $D$ amit eröffnet sich die A ussicht, bisher verschlossene Bereiche des Weltalls durch $\mathrm{G}$ ravitationsw ellenastronomie zu studieren.

\begin{abstract}
W enige Monate stellung der Allgemeinen Relativitätstheorie im N ovember 1915 erkannte Einstein, dass aus seiner Theorie zwangsläufig die Existenz von Gravitationswellen folgt. U nter den vielen stets mit Glanz bestandenen Tests der Allgemeinen Relativitätstheorie ist es besonders diese Voraussage, die noch der Bestätigung durch einen direkten $\mathrm{N}$ achweis harrt.
\end{abstract}

\section{Allgemeine Relativitätstheorie und Gravitationswellen}

Die A llgemeine R elativitätstheorie beschreibt die Gravitation nicht als Kraft, sondern als A spekt der Geometrie des Raum-Zeit-K ontinuums, kurz Raumzeit. Anders als in der $\mathrm{N}$ ewtonschen Physik wird der Raum hier nicht durch eine ebene (E uklidische), sondern durch eine gekrümmte (Riemannsche) $\mathrm{G}$ eometrie beschrieben. $D$ er $R$ aum sel bst ist keine absolute unveränderliche $G$ röße mehr, da er durch die Verteilung von $M$ asse und Energie im U niversum beeinflusst wird. D ie G rundidee der Allgemeinen R elativitätstheorie ist: Die $M$ aterie bestimmt die $K$ rümmung des Raumes, und der Raum bestimmt die Bewegung der $M$ aterie.
$M$ assen rufen eine $K$ rümmung der Raumzeit hervor. Licht und $M$ aterie sind gezwungen, sich entsprechend dieser nicht-euklidischen $M$ etrik zu bewegen. $D$ a sich alle $M$ aterie in Bewegung befindet, ändert sich die $\mathrm{G}$ eometrie der Raumzeit ständig. John A. Wheeler prägte daher den $\mathrm{N}$ amen $\mathrm{G}$ eometrodynamik für E insteins $\mathrm{G}$ ravitationstheorie [1]. 
A nalog zur Elektrodynamik, in der jede beschleunigt bewegte Ladung elektromagnetische Wellen abstrahlt, geben auch beschleunigte $M$ assen $G$ ravitationswellen ab. In Einsteins Theorie sind sie transversal und breiten sich mit Lichtgeschwindigkeit aus. Eine G ravitationswelle äußert sich in einer Verformung der Raumzeit, das heißt in einer Ä nderung $\delta$ I des $A$ bstandes I zwischen zwei Testmassen (A bbildung 1 ). A Is $M$ aß für die Stärke $h$ von $G$ ravitationswellen hat sich das $D$ oppelte der relativen Änderung eingebürgert: $\mathrm{h}=2 \delta \mathrm{l} / \mathrm{l}$. Man nennt diese Größe auch Gravitationswellenamplitude oder einfach Raumdehnung. Da $\mathrm{h}$ eine Amplitude ist, nimmt sie wie die elektrische Feldstärke nur einfach invers mit dem $A$ bstand $D$ von der Q uelle ab, nicht mit $1 / D^{2}$.

Die wesentlichen Eigenschaften einer $G$ ravitationswelle lassen sich aus der Tatsache herleiten, dass es nur M assen eines Vorzeichens gibt. A us diesem $\mathrm{G}$ rund geben erst beschleunigte $M$ assenquadrupole Strahlung ab, während es sich bei elektrischen Ladungen vornehmlich um D ipolstrahlung handelt. D amit hängt auch zusammen, dass die beiden möglichen Polarisationsrichtungen nicht wie im el ektromagnetischen $\mathrm{F}$ all senkrecht aufeinander stehen, sondern sich um $45^{\circ}$ voneinander unterscheiden. Im Teilchenbild hat das masselose $\mathrm{F}$ eldteilchen (G raviton) deshalb den Spin $S=2$, während das Photon $S=1$ hat.

Die von kosmischen Quellen ausgehende Strahlungsleistung kann sehr hoch sein. Bei einer typischen Supernova in der $N$ ähe der M ilchstraße, wie der Supernova 1987A in der $160000 \mathrm{~L}$ ichtjahre entfernten $\mathrm{G}$ roßen M agellanschen Wolke, treffen auf der Erde etwa $10^{31} \mathrm{G}$ ravitonen pro $\mathrm{Q}$ uadratmeter und $\mathrm{Se}$ kunde ein. E in weiteres B eispiel: Zwei einander im A bstand von $100 \mathrm{~km}$ mit einer Periode von 0,01 Sekunden umkreisende $\mathrm{N}$ eutronensterne mit jeweils 1,4 Sonnenmassen im 28000 Lichtjahre entfernten Zentrum der $M$ ilchstraße strahlen eine L eistung von etwa $10^{45} \mathrm{~W}$ ab, was am 0 rt der Erde eine Leistungsdichte von $10^{5} \mathrm{~W} / \mathrm{m}^{2}$ zur Folge hat. $\mathrm{D}$ as entspricht dem $\mathrm{H}$ undertfachen der Strahlungsleistung der Sonne am Ort der Erde. Dennoch bewirkt diese hohe Leistungsdichte nur eine relative Längenänderung von $\delta \mathrm{I} / \mathrm{I} \sim 10^{-18}$. Dies ist eine Folge der geringen Wechselwirkung der Gravitonen mit M aterie. D eshalb kann man G ravitationswellen auch nicht in L aborexperimenten erzeugen und nachweisen. $\mathrm{N}$ ur kompakte kosmische $\mathrm{O}$ bjekte mit großen Beschleunigun- gen und großen Q uadrupolmomenten erzeugen G ravitationswellen mit messbaren A mplituden.

D as Problem der theoretischen A strophysik auf diesem $G$ ebiet ist die Berechnung von $\mathrm{H}$ äufigkeit, Stärke, Wellenform und Frequenz der durch kosmische Ereignisse verursachten G ravitationswellen. D ie mathematische Behandlung ist so kompliziert, dass man auch im Zeitalter der Supercomputer nur grob vereinfachte Modelle durchrechnen kann. Es ist aber gelungen, für eine große Zahl von Q uellen realistische Voraussagen zu machen $[2,3]$.

Das Spektrum der Gravitationswellen umfasst demnach einen weiten $F$ requenzbereich, wobei für denjenigen von $10^{-4}$ bis $10^{4} \mathrm{~Hz}$ ein $\mathrm{N}$ achweis mit heutigen Möglichkeiten aussichtsreich erscheint. Die Beobachtung von G ravitationswellen liefert uns Informationen über die energiereichsten und heftigsten Vorgänge im U niversum sowie über seine frühesten A nfänge. D ieN achweisgrenzefür solche Ereignisse oder $\mathrm{O}$ bjekte reicht, je nach $\mathrm{Fre}$ quenz und Bandbreite der Signale, bis hinunter $z u$ relativen $L$ ängenänderungen $h$ von $10^{-24}$, während von nahen starken Q uellen A mplituden $\mathrm{h}$ bis zu $10^{-18}$ denkbar sind. Wellen ausreichender Stärke erwartet man nicht nur aus unserer $\mathrm{M}$ ilchstraße. $\mathrm{D}$ a die $\mathrm{A}$ mplitude nur gemäß 1/D mit dem Abstand abnimmt, hofft man noch Q uellen im U mkreis von $100 \mathrm{M}$ illionen Lichtjahren beobachten zu können, so dass auch wesentlich häufiger spektakuläre Ereignisse zu erwarten sind. Zum Vergleich: Die A ndromeda-G alaxie ist 2,3 Millionen Lichtjahre entfernt, der große Virgo-G alaxienhaufen $70 \mathrm{M}$ illionen Lichtjahre.

D ie D etails der vorhergesagten E igenschaften der $\mathrm{G}$ ravitationswellen hängen von der zugrunde gelegten Theorie ab. Alternative Theorien der $\mathrm{G}$ ravitation, die mit den sonstigen Beobachtungen übereinstimmen, sagen anders als die Einsteinsche beispielsweise Longitudinalwellen, eine andere $G$ eschwindigkeit (das heißt massebehaftete $G$ ravitonen) oder andere Polarisationen voraus. Der erfolgreiche $\mathrm{N}$ achweis von $\mathrm{G}$ ravitationswellen wird über das Schicksal dieser unterschiedlichen A nsätze entscheiden.

\section{Gravitationswellendetektoren}

Der $\mathrm{N}$ achweis von Gravitationswellen besteht lediglich in einer einfachen $L$ ängenmessung. W ie die Beispiele gezeigt haben, besteht das Problem aber darin, dass die relativen $L$ ängenänderungen $h$ typischerweise nur $10^{-21}$ betragen. A uf den A bstand Erde-Sonne bezogen, entspricht dies dem $D$ urchmesser eines Wasserstoffatoms!

Zur M essung derart kleiner Signale werden im wesentlichen zwei Wege verfolgt. Die ersten Versuche unternahm Ende der 60er J ahre Joseph Weber [4]. Er verwendete Resonanzantennen, große Aluminiumzylinder mit $M$ assen von circa 1,5t. Regt eine G ravitationswelle diesen $K$ örper an, so schwingt er im longitudinalen Grundmodus bei der Eigenfrequenz des Zylinders. Diese Längenänderung sollte durch aufgeklebte Piezokristalle oder die Verstimmung eines aufgesetzten $\mathrm{M}$ ikrowellenresonators gemessen werden.

Frühzeitig erkannte man, dass auch ein Interferometer vom M ichelson-Typ ideal geeignet ist, die von Gravitationswellen erzeugten $L$ ängenänderungen nachzuweisen. Es misst die Phasendifferenz zwischen zwei Lichtwellen, die die unter einem rechten Winkel stehenden Interferometerarme durchlaufen haben. $D$ ies entspricht dem $L$ ängenunterschied beider A rme.

Bis Ende der 90er Jahre war es nicht möglich, die benötigte $D$ etektorempfindlichkeit zu erreichen. Erst die heutige Technik bietet für beide $M$ ethoden $A$ ussicht auf einen erfolgreichen $\mathrm{N}$ achweis von $\mathrm{G}$ ravitationswellen. $\mathrm{Bei}$ den Resonanzantennen hat die Abkühlung auf Temperaturen des flüssigen $\mathrm{H}$ eliums und darunter sowie die Verwendung hochempfindlicher supraleitender Verstärker (SQ U ID s) eine wesentliche Verbesserung gebracht. Durch die Verwendung kugelförmiger $M$ assen ließe sich auch die durch die Zylinderform bedingte Richtungsabhängigkeit dieser $D$ etektoren beseitigen [5].

\section{Gravitationswellenastronomie}

Einen ersten indirekten $\mathrm{H}$ inweis auf die Existenz von $\mathrm{G}$ ravitationswellen gibt es bereits. Die beiden amerikanischen Astrophysiker Russell H ulse und Joseph Taylor entdeckten 1974 ein Sternsystem, in dem sich zwei N eutronensterne umkreisen. Sie studierten dieses Binärsystem mit der Bezeichnung PSR 1913+16 über viele Jahre hinweg und stellten fest, dass sich die beiden $K$ örper langsam einander annähern, wobei sich die U mlaufzeit fortwährend verringert [6]. D ies lässt sich als durch die $A$ bstrahlung von $G$ ravitationswellen bedingter Energieverlust deuten. D ie über 
25 Jahre durchgeführten, sehr genauen Beobachtungen stimmen mit den Voraussagen der Einsteinschen Theorie exakt überein. Dies gilt allgemein als indirekter $\mathrm{N}$ achweis von Gravitationswellen und brachte $\mathrm{H}$ ulse und Taylor 1993 den N obelpreis für Physik ein.

Seitdem richten sich die Bemühungen der theoretischen A strophysiker verstärkt auf die Berechnung der Gravitationsstrahlung von Q uellen, die von den geplanten $D$ etektoren direkt beobachtet werden können. D ie Bilder und $D$ aten, die inzwischen von den großen Weltraumteleskopen geliefert werden, führen zu immer gewagteren Szenarios wie der Vereinigung von superschweren Schwarzen $\mathrm{L}$ öchern bei der $\mathrm{D}$ urchdringung zweier $\mathrm{G}$ alaxien. A ber auch die Strahlung von weniger spektakulären Q uellen lässt sich immer besser berechnen. Dies gibt den Experimentalphysikern eine Vorstellung, auf welche Signale sie sich einstellen müssen. $M$ it Sicherheit wird man aber auch Q uellen und Signale entdecken, an die keiner gedacht hat.

Elektromagnetische Strahlung wird leicht gestreut und absorbiert. Weite Teile des U niversums werden von Dunkelwolken verdeckt und bleiben so der Beobachtung durch klassische astronomische Techniken verborgen. Gravitationswellen dagegen durchdringen solche Wolken ungehindert und werden uns daher völlig neue Einblicke in bisher verschlossene Bereiche des Weltalls liefern. Insbesondere ist mit einer besseren Statistik der Verteilung von $\mathrm{N}$ eutronensternen und Schwarzen Löchern zu rechnen. M an wird dann auch einen genaueren Wert für die mittlere $M$ ateriedichte im $U$ niversum erhalten. A us dieser $\mathrm{G}$ röße ergibt sich, ob das $U$ niversum ewig weiter expandieren oder eines Tages wieder in sich zusammenfallen wird.

Folgende Q uellen lassen sich voraussichtlich mit den geplanten $D$ etektoren beobachten.

Supernovae (vom Typ II) werden durch den Gravitationskollaps eines ausgebrannten Sterns verursacht. D as G leichgewicht im Innern eines Sterns wird dadurch aufrecht erhalten, dass der Strahlungsdruck der G ravitation entgegenwirkt. $\mathrm{N}$ ach dem $\mathrm{E}$ rlöschen der Fusionsvorgänge stürzt der innere $M$ ateriekern im freien Fall in sich zusammen. Wegen der dabei erzeugten hohen Verdichtung kommt es zu einem Rückstoß, der die äußere $H$ ülle sprengt und als $M$ ateriewolke in die U mgebung verteilt. D er K ern stürzt dann, je nach seiner $M$ asse, zu einem $N$ eutronenstern

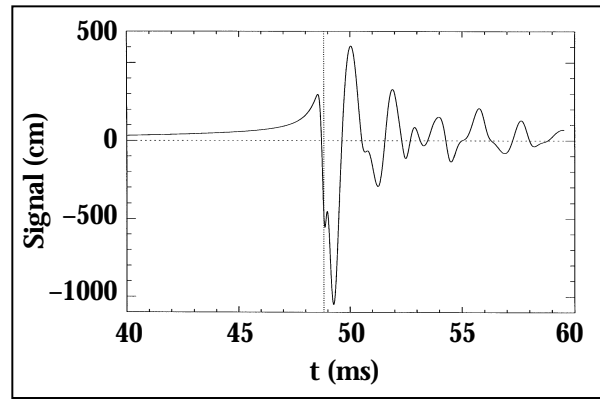

Abb. 2. Berechnetes G ravitationswellensignal eines durch Rotation abgeflachten kollabierenden Sterns. Der Kollaps ge schieht in polarer und äquatorialer $R$ ichtung unterschiedlich schnell (das zeigt die Struktur des starken A usschlags nach unten). Anschließend erfolgt eine gedämpfte O szillation mit zwei verschiedenen Fre quenzen [9].

oder einem Schwarzen Loch zusammen. $\mathrm{H}$ ierbei wird mindestens die Bindungsenergie des $\mathrm{N}$ eutronensterns frei, typischerweise $15 \%$ der Sonnenmasse oder $10^{45} \mathrm{~J}$. D er größte Teil dieser Energie wird in Form von $\mathrm{N}$ eutrinos abgestrahlt, bis zu $1 \%$ als Gravitationswellen, der Rest als elektromagnetische Strahlung.

Die Gravitationswellen werden ungehindert emittiert und liefern I nformationen über Prozesse, die während des Zusammensturzes stattfinden, also $D$ etails des A blaufs der Supernova (A bbildung 2). M an kann mit einem Gravitationswellendetektor der Bildung von $\mathrm{N}$ eutronensternen und Schwarzen Löchern direkt beiwohnen. Die $\mathrm{D}$ auer des $\mathrm{G}$ ravitationswellenpulses beträgt etwa einige hundertstel Sekunden; seine Frequenz liegt bei einigen $100 \mathrm{~Hz}$ bis $1 \mathrm{kHz}$ und die Signalstärke bei bis zu $10^{-18}$, wenn die Supernova in der M ilchstraße stattfindet. Man erwartet dort etwa drei solcher E reignisse pro Jahrhundert. Für die G ravitationswellendetektoren der ersten Generation wird eine $\mathrm{N}$ achweisempfindlichkeit von $10^{-21}$ angestrebt, so dass man auch Supernovae im benachbarten Virgo-G alaxienhaufen beobachten kann. Wenn nur $1 \%$ aller Sternzusammenbrüche Signale ausreichender Stärke erzeugt, dann sollte man mehrere E reignisse pro J ahr sehen.

A ußerdem rotiert ein $\mathrm{N}$ eutronenstern be seiner Entstehung zunächst mit etwa 200 U mdrehungen pro Sekunde und bildet irreguläre $M$ assenverteilungen aus, die resonante Schwingungsmoden anregen. D ie Energie in einer solchen Mode kann der beim Kollaps freigesetzten vergleichbar sein, so dass sie sich dem Signal aufprägt. Ist der Stern etwa infolge der Rotation abgeplattet, so wird man die schnelleren polaren Schwingungen von den langsameren äquatorialen unterscheiden können (A bbildung 2). Solche Resonanzmoden werden in einigen zehntel Sekunden abklingen. Die Frequenz wird hauptsächlich durch die Modenfrequenz bestimmt, nicht durch die R otation.

Verschmelzende D oppel systeme aus N eutronensternen oder Schwarzen L öchern gehören zu den wichtigsten $\mathrm{Q}$ uellen für erdgebundene Interferometer. Ein solches System gibt, wie in dem Fall des Binärpulsars PSR 1913+16, Energie in Form von G ravitationswellen $a b$, die Partner rücken sich dadurch immer näher, wobei sie sich immer schneller umkreisen, bis sie schließlich ineinander stürzen (A bbildung 3). D ie von innen bis kurz vor der Verschmelzung abgestrahlte Wellenform ist theoretisch sehr gut bekannt. Das macht es möglich, selbst schwache Signale aus dem sie überdeckenden Rauschen herauszufiltern. Typische Frequenzen liegen im Bereich von 10 bis $100 \mathrm{~Hz}$; typische A mplituden sind $10^{-21}$ bis $10^{-22}$. Signal stärken von $10^{-}$ 23 und kleiner sollten noch nachweisbar sein. Zwei $\mathrm{N}$ eutronensterne ließen sich daher bis zu einer Entfernung von $10^{9} \mathrm{~L}$ ichtjahren beobachten. Die Ereignisrate für die spektakulären Sternverschmelzungen ist noch etwas unsicher; bei reinen $\mathrm{N}$ eutronensternsystemen rechnet man mit nur einer beobachtbaren Verschmelzung pro Jahr. Für die weiterentwickelten $D$ etektoren der zweiten $G$ eneration könnten es aber sogar einige hundert $\mathrm{Be}$ obachtungen pro Jahr sein.

Der F requenzverlauf des G ravitationswellensignals kann so detailliert modelliert werden, dass sich die beteiligten $M$ assen und $B$ ahnparameter bestimmen lassen, insbesondere auch der gegenseitige $A$ bstand. A us der tatsächlich gemessenen A mplitude $h$ kann man dann die Entfernung bis zur Q uelle ermitteln. D ies in Verbindung mit Messungen der optischen Rotverschiebung bietet die genaueste $M$ öglichkeit, die $\mathrm{H}$ ubble-K onstante und damit das A Iter des U niversums zu bestimmen [7].

Schnell rotierende Neutronensterne, die in zwei entgegengesetzte Richtungen Strahlung abgeben, nennt man Pulsare. Sie können G ravitationswellen abstrahlen, wenn ihre G estalt von der A xialsymmetrie abweicht. D as kann durch „Beulen“ verursacht werden, die beim Kollaps zum N eutronenstern „eingefroren“ sind, oder durch dynamische Vorgänge wie der A nregung von Eigenschwingungen. Der Energieverlust hängt dabei von der Elliptizität $\mathrm{e}=1-\mathrm{b} / \mathrm{a}$ des Sterns ab ( $\mathrm{a}$ ist die große 

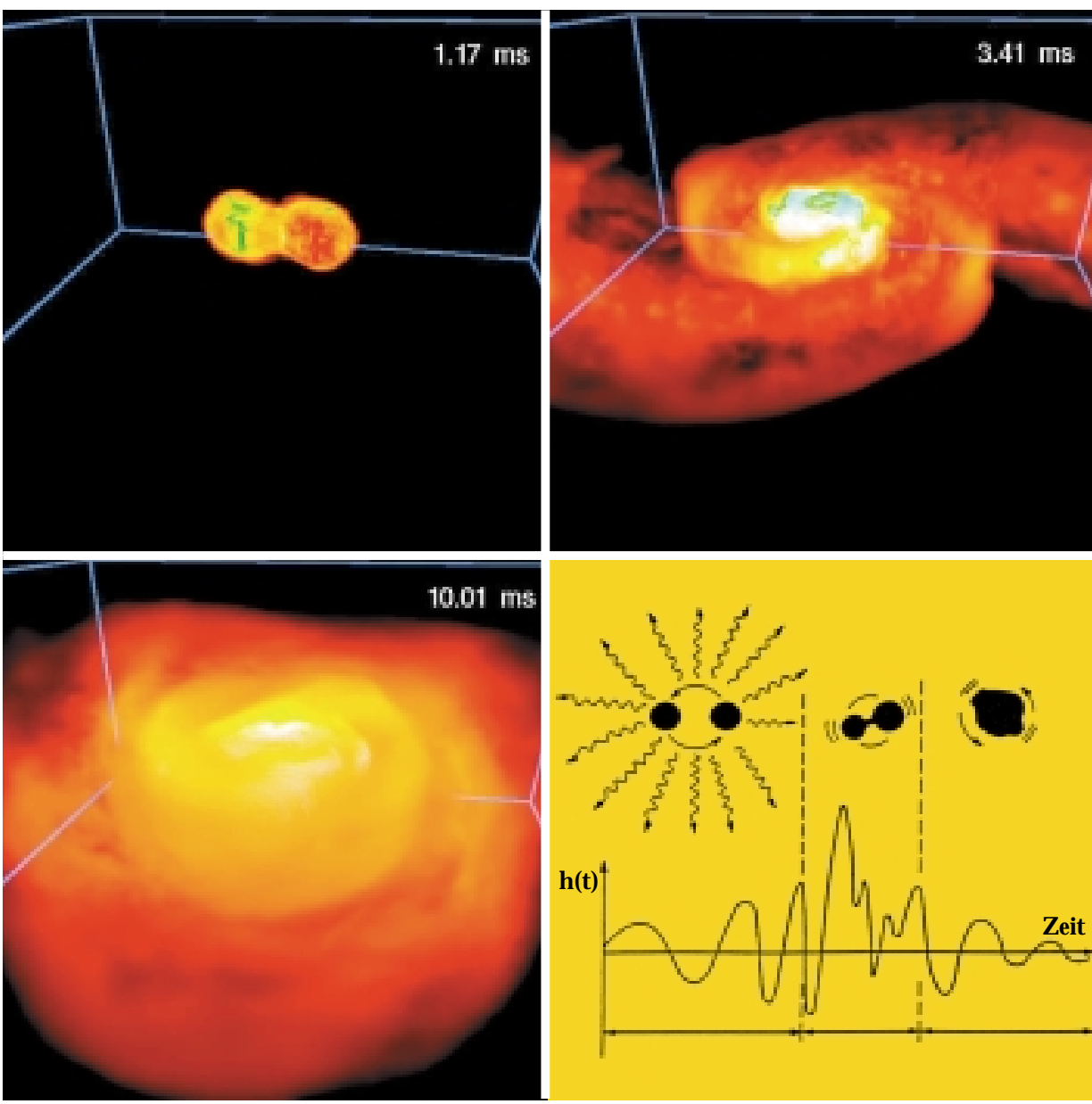

Abb. 3. Computersimulation zweier verschmelzender Neutronensterne In Bruchteilen einer Sekunde gehen die beiden $\mathrm{N}$ eutronensterne in einem $10^{8} \mathrm{G}$ rad heißen Feuerball auf. Man achte auf die Zeitskala! R echts unten: Schematische $D$ arstellung der Verschmelzung zweier einander umkreisender kompakter 0 bjekte und der daraus resultierenden $\mathbf{G}$ ravitationswelle (R uffert, J anka, M PI für Astrophysik, G arching).

und $\mathrm{b}$ die kleine $\mathrm{H}$ albachse der Ellipse). Bereits eine Elliptizität um $10^{-8}$ reicht aus, um beobachtbare Strahlungsamplituden zu erzeugen. Typische Rotationsfrequenzen sind 10 bis $1000 \mathrm{H}$ z. M an erwartet ein nahezu periodisches Signal mit der doppelten Rotationsfrequenz, die nur durch die Präzession des Sterns und die allmähliche A bnahme der Rotationsgeschwindigkeit modifiziert wird.

Ein N eutronenstern kann durch seine $\mathrm{G}$ ravitation von einem nahe gelegenen normalen Stern $M$ aterie abziehen, die in Form einer A kkretionsscheibe auf inn übergeht und seine R otation beschleunigt (A bbildung 4). Solche akkretierenden $\mathrm{N}$ eutronensterne bilden das Zentralobjekt in den meisten binären Röntgenquellen in der Galaxis. Sie können auch intensive kontinuierliche $\mathrm{G}$ ravitationswellen aussenden, wenn in einem hinreichend schnell rotierenden $\mathrm{N}$ eutronenstern instabile Eigenmoden angeregt werden oder wenn Rotationsachse des Sterns und A kkretionsachse nicht übereinstimmen und beide umeinander präzedieren. D ie G ravitationsw ellenfrequenz entspricht dann praktisch der Präzessionsfrequenz.

Der U rknall sollte nach allen gängigen kosmologischen Modellen einen $\mathrm{H}$ intergrund von $G$ ravitationsstrahlung erzeugt haben. U m ihn nachzuweisen, muss man die M essdaten zweier unabhängiger D etektoren miteinander vergleichen. Wenn beide nicht zu weit voneinander entfernt sind, besteht eine enge Korrelation ihrer M essdaten, so dass sich der $\mathrm{H}$ intergrund ermitteln lässt. $\mathrm{A}$ us der spektralen Energiedichte dieses Rauschens ließe sich ebenfalls eine A ussage über die $M$ ateriedichte des $U$ niversums gewinnen.

Da die gravitative $\mathrm{H}$ intergrundstrahlung Informationen enthält, die aus einer Zeit von $10^{-35}$ Sekunden nach der Entstehung des $U$ niversums stammen, bietet sie die M öglichkeit, die A ussagen der verschiedenen kosmologischen Modelle über die Kosmogenese $z u$ überprüfen. Der zur Zeit aussichtsreichste $K$ andidat für eine vereinheitlichte Theorie aller Wechselwirkungen ist die Superstring-

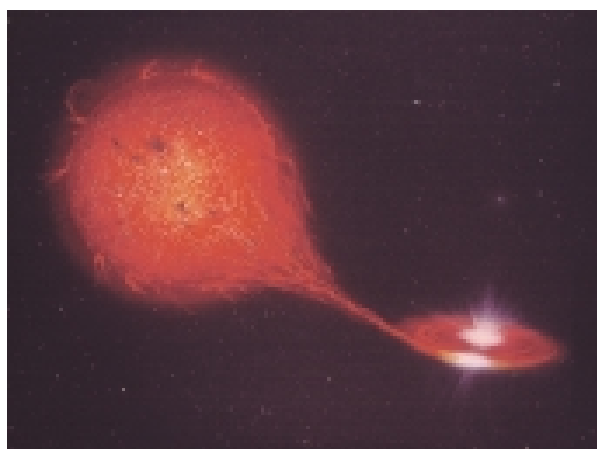

Abb. 4. Darstellung eines Doppelsystems, bei dem Materie von einem normalen Stern zum Neutronenstern hinüberfließt und sich dort in einer Scheibe anordnet.

Theorie. $\mathrm{N}$ ach ihr gelten topologische $\mathrm{De}$ fekte (Strings), die bei Phasenübergängen im frühen U niversum entstanden sein könnten, als $\mathrm{H}$ auptquelle der $\mathrm{H}$ intergrundstrahlung. Die Theorie sagt ein bestimmtes Spektrum des $\mathrm{G}$ ravitationswellenhintergrundes voraus. Während sich die A ussagen der String-Theorie mit $\mathrm{H}$ ilfe von Superbeschleunigern (aus finanziellen Gründen) nur in begrenztem $M$ aßse testen lassen, werden die geplanten G ravitationswellendetektoren in der Lage sein, diese Vorhersage zu überprüfen.

A us zahlreichen astronomischen Beobachtungen muss man schließen, dass sich im Zentrum fast jeder $\mathrm{G}$ alaxie ein superschweres Schwarzes $L$ och befindet, das eine $M$ asse entsprechend $10^{6}$ bis $10^{9}$ Sonnen enthält. Solche $O$ bjekte strahlen während ihrer Entstehung oder beim Verschlucken von kompakten Sternen und kleineren Schwarzen Löchern Gravitationswellen im niederfrequenten $\mathrm{Be}$ reich $\left(10^{-4}\right.$ bis $\left.1 \mathrm{H} \mathrm{z}\right)$ aus. $D$ as spektakulärste Ereignis in der $\mathrm{G}$ ravitationswellenastronomie überhaupt wäre die Verschmelzung zweier superschwerer Schwarzer Löcher beim Zusammenstoß zweier Galaxien. Wegen der großen Signalamplitude (man erwartet, dass das Signal um bis zu einem Faktor $10^{4}$ über dem Rauschen liegt) lässt sich die Position der beiden $G$ alaxien sehr genau bestimmen, so dass man unter Zuhilfenahme optischer $\mathrm{D}$ aten die $\mathrm{H}$ ubble-K onstante und den $\mathrm{Ab}$ bremsparameter des Weltalls auf $1 \%$ genau auswerten kann. A uch daraus ließe sich auf die M assendichte des $U$ niversums schließen.

\section{Laserinterferometer}

Zur Erschließung dieses völlig unerforschten weiten Feldes der Gravitationswellenastronomie sollen $\mathrm{L}$ aserinterferometer in $\mathrm{M}$ ichelson-A nordnung eingesetzt werden. $E$ in solches Instrument vergleicht die durch eine $G$ ravitationswelle hervorgerufene Raumdehnung und -stauchung in zwei senkrecht auf- 
einander stehenden Richtungen. D as Prinzip ist in A bbildung 5 dargestellt: Strahlteiler und Spiegel dienen als Testmassen, ein Laser als Lichtquelle; gemessen wird die Phasendifferenz zwischen den beiden Teilstrahlen. $D$ ie M essung erfolgt mit einer $\mathrm{N}$ ullmethode: $\mathrm{D}$ as Interferometer wird so voreingestellt, dass am Ausgang Dunkelheit herrscht (Interferenzminimum). Die durch die Armlängenänderungen verursachten $\mathrm{H}$ elligkeitsschwankungen werden mit einer Photodiode registriert.

Da die zu messende $G$ röße $\delta$ l proportional zu h.l ist, sollte man den Lichtweg I offenbar möglichst groß machen. Das 0 ptimum ist aber erreicht, wenn er gleich der halben Wellenlänge der $\mathrm{G}$ ravitationswelle ist, denn in der nächsten $\mathrm{H}$ albwelle kehrt sich der Effekt um. Für eine $G$ ravitationswellenfrequenz von $1 \mathrm{kH}$ z ergibt sich diese L änge zu $150 \mathrm{~km}$. D er Bau eines Interferometers mit einer derartigen Armlänge ist offensichtlich nicht realisierbar. Glücklicherweise kann man den Lichtweg durch ein Paar von Spiegeln falten, so dass man Detektoren mit A rmlängen von "nur" einigen hundert oder tausend $M$ etern bauen muss.

A nfang der 70er Jahre wurden erste Versuche durchgeführt, ein Laserinterferometer für den $\mathrm{G}$ ravitationswellennachweis einzusetzen. Zunächst wurde an kleineren Prototypen mit A rmlängen von 3 bis $40 \mathrm{~m}$ vor allem studiert, welche physikalischen Prozesse die E mpfindlichkeit bei den verschiedenen Frequenzen begrenzen. D ies führte zu einer A nalyse der möglichen Rauschquellen und zu den A nforderungen, die an die Konstruktion gestellt werden müssen, um die gewünschte $\mathrm{Emp}$ findlichkeit zu erreichen.

\section{Störsignale und Rauschquellen}

Beim Einsatz von Laserinterferometern für den $\mathrm{G}$ ravitationswellennachweis treten viele Störungen auf, die zu Schwankungen in der Lichtwegdifferenz zwischen den beiden A rmen führen und dadurch $\mathrm{G}$ ravitationswellensignale vortäuschen. Um den erfolgreichen Betrieb von Gravitationswellendetektoren überhaupt erst zu ermöglichen, musste eine Reihe technischer Verbesserungen und $\mathrm{N}$ euentwicklungen durchgeführt werden, da die Anforderungen den vorhandenen Standard auf fast allen $\mathrm{G}$ ebieten überstiegen. Im folgenden sei die Lösung einiger Probleme am Beispiel unseres deutsch-britischen Projekts GEO 600 geschildert.

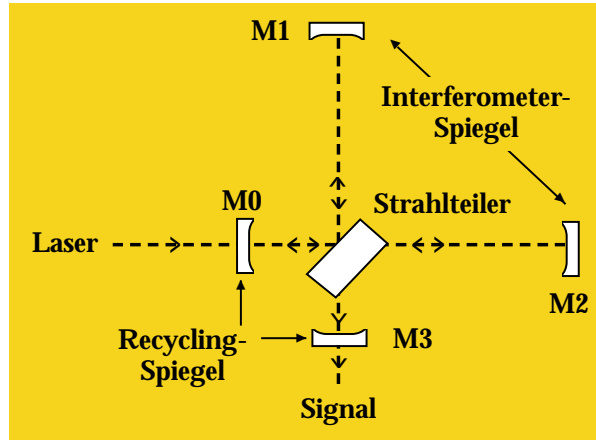

Abb. 5. Michelson-Interferometer mit doppeltem R ecycling. D er Laserstrahl fällt von links ein und trifft auf einen Strahlteiler. M1 und M 2 sind die beiden Endspiegel in den Interferometerarmen, MO dient zur Leistungsrückführung, M3 zur Signalverstärkung.

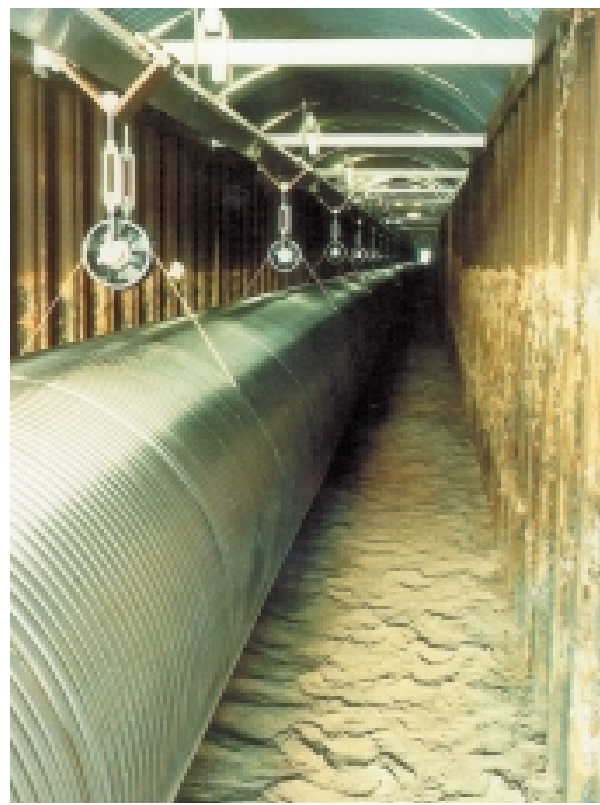

A bb. 6. A ufhängung des Vakuumrohrs beiGEO 600 .

Eine wesentliche Q uelle von Störungen stellen thermische und mechanische Fluktuationen der L uftdichte dar. Alle optischen A ufbauten ( $L$ aser, Strahlteiler, Spiegel, $N$ achweisoptik) werden daher in großen Vakuumtanks untergebracht; die M essstrecke selbst verläuft in evakuierten Rohren mit einem Durchmesser von $60 \mathrm{~cm}$ (A bbildung 6). Das Vakuum muss besser als $10^{-11}$ bar sein, so dass man sich schon im Bereich des UItrahochvakuums befindet. $D$ as macht spezielle Reinigungstechniken und das Ausheizen des Rohrs und der Komponenten der A nlage erforderlich.

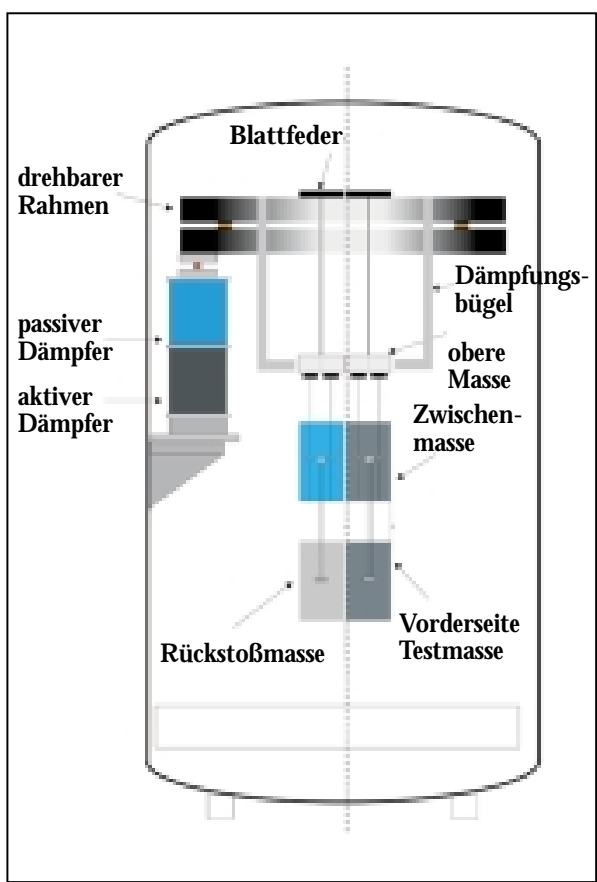

Abb. 7. Die Hauptspiegel von GEO 600 werden durch aktive und passive Schwingungsdämpfer von seismischen Störungen isoliert. D rei um $120^{\circ}$ versetzte Dämpfer (im Längsschnitt ist nur einer links zu se hen) tragen einen Rahmen, an dem über zwe Blattfedern eine größere Zwischenmasse hängt. An dieser ist wiederum über vier Blattfedern ein D oppelpendel aufgehängt. Es besteht aus einer weiteren $Z$ wischenmasse und dem eigentlichen Spiegel, der die Testmasse darstellt.

$\mathrm{N}$ aheliegende Störquellen für einen G ravitationswellendetektor sind Bodenerschütterungen aller A rt. Siekönnen beispielsweise durch die Vibrationen der Vakuumpumpen hervorgerufen werden, durch die M eeresdünung oder durch Fahrzeuge, die sich an der A nlage vorbei bewegen. Die $\mathrm{H}$ auptquelle stellt die seismische Bodenunruhe dar, die mit $1 / f^{2} a b$ fällt. Sie sorgt für A mplituden, die um viele Zehnerpotenzen über den zu messenden liegen. E ine wesentliche A ufgabe stellte daher die Entwicklung einer wirkungsvollen Vibrationsisolation dar, die verhindert, dass sich die Schwingungen aus der U mgebung auf die im Tank aufgehängten optischen K omponenten übertragen.

Die Schwingungsdämpfung für die $\mathrm{H}$ auptspiegel von GEO 600 erfolgt in mehreren Stufen (A bbildung 7); sie enthält aktive und passive Elemente. Im aktiven Teil werden die Schwingungsamplituden mit G eophonen ge- 
messen und durch Piezoaktuatoren weitgehend kompensiert. Der passive Teil besteht aus zweilagigen $D$ ämpfern aus $M$ etall und Gummi, wodurch mittlere und hohe Frequenzen abgeblockt werden. Die A ufhängung der optischen Komponenten erfolgt in Form eines Mehrfachpendels. H ierdurch wird eine sehr wirkungsvolle Filterung von Frequenzen erreicht, die oberhalb der Eigenfrequenz des Pendels liegen. D iese lässt sich durch die Wahl der Pendellänge beeinflussen. Ein Pendel mit einer $L$ änge von $1 \mathrm{~m}$ hat die Eigenfrequenz $f_{0}=0,5 \mathrm{~Hz}$. Bei einer Beobachtungsfrequenz $f=500 \mathrm{~Hz}$ erfolgt dann schon bei einem Einfachpendel eine $\mathrm{H}$ erabsetzung der Störamplituden um den Faktor $\left(f_{0} / f\right)^{2}=10^{6}$. D ie erwünschte $G$ üte $Q$ (das Inverse des $D$ ämpfungsfaktors) von etwa $10^{8}$ erreicht man durch eine monolithische A ufhängung, indem man die Q uarzspiegel an angeschmolzenen Q uarzfasern aufhängt.

Zu kleinen Frequenzen hin wird die Schwingungsisolierung immer aufwendiger. Es wird daher allgemein nicht möglich sein, mit erdgebundenen Detektoren Frequenzen zu beobachten, die unterhalb von einigen $\mathrm{Hz}$ liegen, bei GEO 600 unterhalb von einigen zehn $\mathrm{Hz}$.

Die Wärmebewegung der A tome führt zur A nregung von Eigenmoden in den optischen Komponenten, beispielsweise zu Dickenoder Biegeschwingungen. Die Spiegeloberfläche bewegt sich dabei vor und zurück, so dass ein Gravitationswellensignal vorgetäuscht wird. Solche internen Störungen lassen sich nicht abschirmen. $\mathrm{H}$ iergegen hilft nur, alle Spiegelresonanzen weit außerhalb des gewünschten Frequenzbereichs zu verlegen, etwa oberhalb von $5 \mathrm{kH}$ z. D ie niederfre- quenten A usläufer der Störungen nehmen mit der Spiegel masse und ihrer mechanischen G üte $\mathrm{Q}$ ab. D urch Wahl eines geeigneten $\mathrm{M}$ aterials ( $Q$ uarzglas, $Q \sim 10^{7}$ ) und einer speziellen zylindrischen Form mit einem Verhältnis von D urchmesser zu Dicke von 2:1 lässt sich die Amplitude der thermischen Bewegung hinreichend unterdrücken. D ie naheliegende Lösung einer Abkühlung der optischen Komponenten ist für die derzeitigen $D$ etektoren nicht vorgesehen. $\mathrm{D}$ a die thermische A mplitude proportional zur Wurzel aus der Temperatur $\mathrm{T}$ ist, wird das erst unterhal b von $4 \mathrm{~K}$ interessant, erfordert also $\mathrm{K}$ ühlung mit flüssigem $\mathrm{H}$ elium. $\mathrm{D}$ as ist mit einem vertretbaren A ufwand derzeit nicht zu machen und wegen der hohen L ichtleistung auch nicht zu halten.

A $n$ das M aterial der Spiegel und des Strahlteilers sowie an die Q ualität der darauf aufgebrachten hochreflektierenden Schichten werden ebenfalls höchste A nforderungen gestellt. Jede Wellenfrontverzerrung durch unvollkommene optische Elemente stört die Interferenz. Gestreutes Licht kann an den nicht schwingungsgedämpften $W$ änden reflektiert werden und erzeugt bei der Überlagerung mit dem $\mathrm{H}$ auptstrahl ein Störsignal. D ie M ikrorauhigkeit der Substrate darf daher nur in der $\mathrm{G}$ rößenordnung von $1 \AA$ liegen, und das über eine Spiegelabmessung von etwa $20 \mathrm{~cm}$. Die Technik der L eistungsüberhöhung (siehe unten) setzt Komponenten mit extrem kleinen Verlusten voraus. Für den Strahlteiler wurde in Zusammenarbeit von GEO 600 und dem französisch-italienischen Projekt VIR$\mathrm{GO}$ durch die Firma $\mathrm{H}$ eraeus ein besonders homogenes Q uarzglassubstrat entwickelt, das A bsorptionsverluste von lediglich einigen $10^{-6}$ pro Zentimeter aufweist. Dadurch wird

Tabelle 1: D ie im Bau befindlichen G ravitationswellendetektoren.

\begin{tabular}{|c|c|c|c|c|}
\hline Projekt & & $\begin{array}{l}\mathrm{G} \\
\mathrm{E} \\
\mathrm{O}\end{array}$ & & $((O)))$ \\
\hline Ort & $\begin{array}{l}\text { M itaka } \\
\text { (Japan) }\end{array}$ & $\begin{array}{l}\mathrm{H} \text { annover } \\
\text { (D eutschland) }\end{array}$ & $\begin{array}{l}\text { H anford } u . \\
\text { Livingston (U SA ) }\end{array}$ & $\begin{array}{l}\text { Pisa } \\
\text { (I talien) }\end{array}$ \\
\hline A rmlänge & $300 \mathrm{~m}$ & $600 \mathrm{~m}$ & je $4 \mathrm{~km}$ & $3 \mathrm{~km}$ \\
\hline Kosten & $20 \mathrm{M}$ io. D M & $10 \mathrm{M}$ io. D M & $450 \mathrm{M}$ io. DM & $150 \mathrm{M}$ io. D M \\
\hline M essbeginn & 2000 & 2001 & $2001 / 02$ & 2002 \\
\hline
\end{tabular}

einelokaleE rwärmung der Spiegel und damit die A usbildung thermischer L insen im Strahlengang des $L$ ichtes vermieden.

\section{Photonen-Recycling}

A Is Lichtquelle des Interferometers benötigt man einen Dauerstrichlaser mit hoher Ausgangsleistung in einer stabilen transversalen und longitudinalen Mode, der monatelang stabil arbeitet. D as L aser Zentrum $\mathrm{H}$ annover hat speziell für das GEO - Projekt einen solchen Laser entwickelt. Es handelt sich dabei um einen mit $L$ aserdioden gepumpten monolithischen $M$ iniatur- $N$ d:YAG-Ringlaser als Strahlungsquelle mit $2 \mathrm{~W}$ A usgangsleistung im Einmodenbetrieb. D iese Strahlung wird in einen laserdiodengepumpten YA G -Stab eingekoppelt (Injection Locking) und auf $10 \mathrm{~W}$ verstärkt. $D$ as $L$ aserlicht wird dann phasenmoduliert, um Regelsignale für die verschiedenen F reiheitsgrade des I nterferometers ableiten zu können.

Eine fundamentale Empfindlichkeitsgrenze für M essungen mit Licht bildet das Schrotrauschen. Photodioden wandeln die L ichtleistung am Ausgang des Interferometers in Strom um. Die statistischen Schwankungen des Photonenflusses $n$ rufen ein weißes $\mathrm{R}$ auschen im Photostrom hervor, das entsprechende Schwankungen der optischen Wegdifferenz vortäuscht: $\delta \mid \sim 1 / \sqrt{n} \sim 1 / \sqrt{\mathrm{P}}$. H iernach ließe sich die $G$ enauigkeit der R egistrierung beliebig verbessern, je größer die zur Verfügung stehende $L$ ichtleistung $P$ ist.

Dem steht jedoch ein anderer Effekt entgegen: der Strahlungsdruck, den das Laserlicht auf die Endspiegel ausübt. Die statistische Schwankung der Photonenzahl erzeugt ein entsprechendes Schwanken in der Position der Spiegel und täuscht damit ein Signal vor. Dieser Einfluß wächst mit zunehmender Lichtleistung an. D ie optimale L eistung liegt dann vor, wenn die durch das Schrotrauschen und den Strahlungsdruck vorgetäuschten Signale gleich groß sind. Für GEO 600 betrüge sie etwa $1 \mathrm{MW}$. D as ist natürlich weit mehr L eistung als jeder verwendbare $L$ aser liefert. Es gibt jedoch eine $M$ ethode, die im Interferometer befindliche $L$ ichtleistung merklich zu vergrößern: das „Power Recycling“ (Leistungsrückführung). Sie ist in allen großen D etektoren vorgesehen.

Beim Power Recycling wird die im Interferometer umlaufende Lichtleistung erhöht, indem man sie mehrfach verwendet. Dabei 
macht man sich zunutze, dass der A usgang des Interferometers durch einen Regelkreis dunkel gehalten wird. Bei einer solchen "destruktiven" Interferenz wird jedoch kein Licht vernichtet, sondern nur umverteilt. In dieser Betriebsart verhält sich das Interferometer wie ein Spiegel: A lles Licht geht zurück zum Eingang. Durch einen zusätzlichen Spiegel kann dieses Licht erneut in das I nterferometer eingespeist werden. Der Zusatzspiegel und das gesamte Interferometer müssen dabei einen optischen Resonator bilden. In Resonanz lässt sich die Lichtleistung um einen Faktor erhöhen, der gleich dem Inversen der Verluste ist. Beim Garchinger GEO-Prototyp konnte so eine Vergrößerung der umlaufenden Lichtleistung um einen Faktor 300 erreicht werden.

D as Signal-Recycling sorgt mit einer ähnlichen $M$ ethode für eine Ü berhöhung des Signals. Eine Gravitationswelle prägt ihre Frequenz der des umlaufenden Laserstrahls auf. Dies führt zu einer Phasenmodulation des Laserlichts, d. h. zu Seitenbändern im Abstand der Gravitationswellenfrequenz links und rechts von der $L$ ichtfrequenz des $L$ asers. Diese Seitenbänder enthalten die eigentliche Information. D ie Phasendifferenz der Träger in den beiden A rmen wird auf $180^{\circ}$ gehalten, so dass dieses Licht zum Eingang zurückläuft: Darauf basiert die Leistungsüberhöhung. Die Seitenbänder werden dagegen in den beiden A rmen in A ntiphase erzeugt; sie verlassen das Interferometer am A usgang. Ein weiterer Spiegel an dieser Stelle bildet mit dem Interferometer einen optischen Resonator, in dem die Seitenbänder resonant überhöht werden. A bhängig von der R eflektivität dieses Spiegels lässt sich die Bandbreite des D etektors einstellen: entweder breitbandig, um Gravitationswellen in einem Bereich bis zu einigen $\mathrm{kHz}$ zu untersuchen, oder aber sehr schmalbandig (einige $\mathrm{H} z$ ), wenn die Frequenz der $\mathrm{Q}$ uelle bekannt ist.

Je schmalbandiger der Detektor, um so größer wird seine Empfindlichkeit. Durch eine kleine Änderung der Spiegelposition lässt sich der Detektor außerdem auf be stimmte Gravitationswellenfrequenzen abstimmen. D arüber hinaus führt dieses Verfahren zu einer erheblichen Verbesserung des optischen K ontrastes: G estreutes L icht befindet sich nicht in Resonanz mit dieser A nordnung und wird daher nicht wie das Signal überhöht. A $m$ G archinger GEO -Prototypen wurde bereits eine zehnfache Signalüberhöhung erzielt [8].

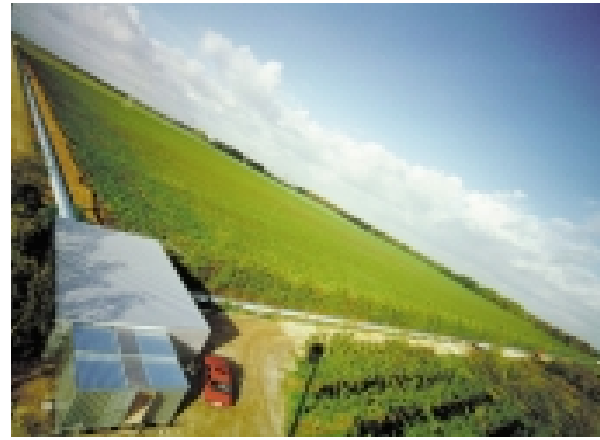

A bb. 8. D as südlich von $H$ annover gelegene GEO 600. Man sieht die beiden $600 \mathrm{~m}$ langen Gräben, in denen das Vakuumrohr aufgehängt ist. Im Zentralhaus (vorn) be finden sich Laser, Strahlteiler und Signalaufnahme, in den beiden kleineren $\mathrm{Ge}$ bäuden (links und rechts) die Endspiegel des Interferometers.

\section{Ein weltweites Netz von Detektoren}

In den letzten Jahren hat man weltweit mit dem Bau großer Laserinterferometer für den $\mathrm{N}$ achweis von $\mathrm{G}$ ravitationswellen begonnen (Tabelle 1). Im Rahmen des U S-amerikanischen Projekts LIGO werden Detektoren mit $4 \mathrm{~km}$ langen A rmen gleich an zwei Standorten errichtet: einen im $\mathrm{N}$ ordwesten der USA (Washington) und einen im Südosten (L ouisiana). D adurch sind die Wissenschaftler der USA autark: Sie können sich in K oinzidenzmessungen gegenseitig verifizieren.

Von ähnlicher Größe ist das bei Pisa entstehende französisch-italienische Projekt VIRGO . Ebenso wie LIGO arbeitet VIRGO mit Fabry-Pérot-Resonatoren in den A rmen und verwendet die Technik der Leistungsrückführung. Als Spezialität bietet VIRGO eine besonders aufwendige seismische I solierung: eine $K$ ette von fünf hintereinander geschalteten Pendeln mit zusätzlicher Verwendung von magnetischen A ntifedern soll $M$ essungen bis in den wissenschaftlich sehr interessanten Frequenzbereich von $10 \mathrm{~Hz}$ ermöglichen.

Gemeinsam von Großbritannien und Deutschland gebaut wird GEO 600 mit $600 \mathrm{~m}$ A rmlänge (A bbildung 8). Federführend sind Wissenschaftler aus $G$ arching, $\mathrm{H}$ annover, Glasgow, Cardiff und Potsdam. Die durch die kürzere $A$ rmlänge verminderte Empfindlichkeit lässt sich durch gleichzeitiges Power- und Signal-Recycling weitgehend kompensieren. Bei der L eistungsrückführung wird ein Ü berhöhungsfaktor von 1000 angestrebt. D ie bisher nur bei GEO 600 verwendete Technik der Signalüberhöhung gleicht besonders bei höheren Frequenzen die kürzere A rmlänge aus.

A $m$ weitesten fortgeschritten ist das kleinste der Interferometer, TAMA 300, das mit $300 \mathrm{~m}$ A rmlänge in Japan gebaut wird. Die optische Konzeption lehnt sich eher an die LIGO - und VIRGO-D etektoren an, allerdings nicht mit dem A nspruch, in der Empfindlichkeit mit diesen mithalten zu können. TAM A 300 zielt in erster Linie auf die Entwick lung und E rprobung neuer K onzepte ab, diefür den A usbau der künftigen großen $D e$ tektoren benötigt werden.

D ie verschiedenen Projekte stehen nicht etwa im Wettbewerb miteinander, im Gegenteil: Sie sind auf einander angewiesen. Die D aten eines einzelnen $D$ etektors haben für pulsförmige Signale, beispielsweise von Supernovae, keine besondere A ussagekraft. Erst in K oinzidenz mit einem weit entfernten Detektor kann man sicher sein, nicht lokalen Störungen aufgesessen zu sein. Daher bilden zwei Detektoren das absolute M inimum, um die bloße Existenz von Gravitationswellen zu beweisen. $U \mathrm{~m}$ aber auch Informationen über die Richtung der Quelle sowie über Zeitstruktur und Polarisation der Signale zu erhalten, benötigt man ein weltweites $\mathrm{N}$ etz von wenigstens vier $D$ etektoren. Wenn alles gut geht, existiert dieses $\mathrm{N}$ etz zu Beginn des nächsten Jahrzehnts. D ie Jahrtausendwende markiert dann auch den Beginn des Zeitalters der G ravitationswellenastronomie. Den A rbeitsbereich dieser neuartigen Teleskopezeigt A bbildung 9 .

\section{USA - ein Detektor im Weltall}

Leider wird der Frequenzbereich unterhalb von $10 \mathrm{~Hz}$ für Detektoren auf der Erde wegen des störenden E influsses der Seismik und von $\mathrm{G}$ ravitationsfeldern bewegter $\mathrm{O}$ bjekte wohl für immer verschlossen bleiben. Dabei senden einige der spektakulärsten Q uellen von $\mathrm{G}$ ravitationswellen, wie superschwere Schwarze Löcher mit M illionen Sonnenmassen, Signale im M illihertzbereich aus. Diesen Frequenzen entsprechen Wellenlängen von einigen Millionen Kilometern. Detektoren mit geeigneten A rmlängen lassen sich auf der Erde nicht realisieren - wohl aber im Weltraum. D as ist das Ziel der LISA-M ission, die von der Europäischen Weltraumbehörde 


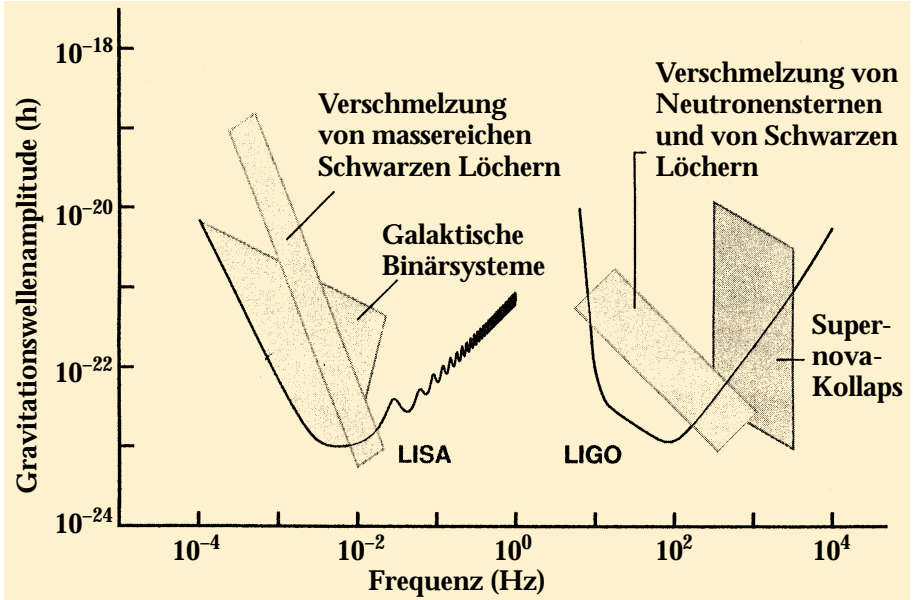

A bb. 9. E mpfindlichkeitskurven des geplanten Weltraumdetektors LISA und eines der im Bau befindlichen erdgebundenen D etektoren (LIG 0 ) sowie Signalstärken und Frequenzbereiche einiger typischer $Q$ uellen von G ravitationswellen.

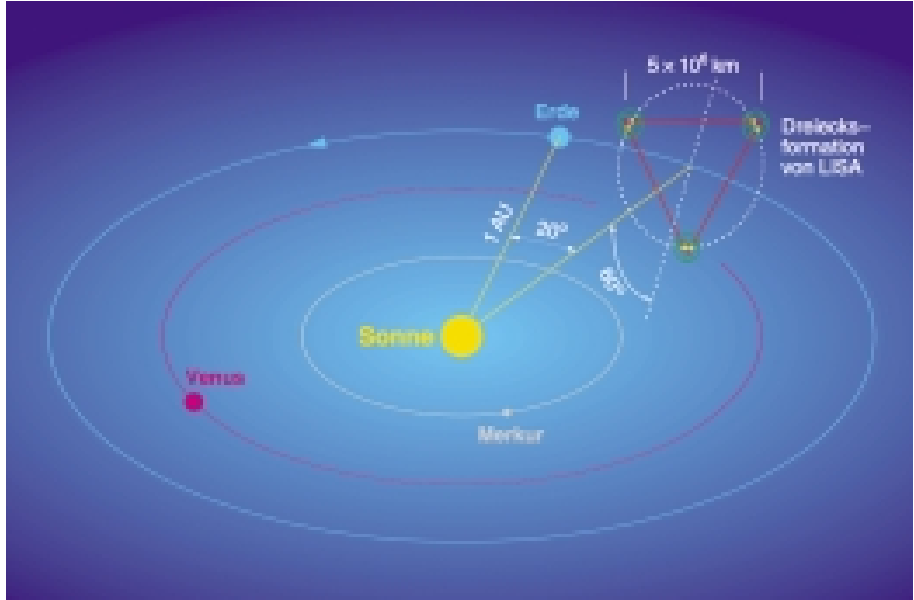

A bb. 10. D ie U mlaufbahnen der drei LISA-Satelliten.
ESA im Rahmen ihres wissenschaftlichen Zukunftsprogramms für einen Start ab 2009 ausgewählt wurde, und die parallel dazu in den U SA durch die N A SA eingehend untersucht wird.

Bei LISA (L aser Interferometer Space A ntenna) sind drei identische Satelliten in Form eines gleichseitigen $D$ reiecks angeordnet, das um $20^{\circ}$ hinter der Erde versetzt entlang der Erdbahn um die Sonne kreist (A bbildung 10 und auf Seite 14). Die Satelliten bilden zusammen ein Laserinterferometer mit $5 \mathrm{Mil}$ lionen $\mathrm{km}$ A rmlänge und einem Winkel von $60^{\circ}$ zwischen den A rmen. Jeder Satellit enthält einen Infrarotlaser mit $1 \mathrm{~W}$ A usgangsleistung. Wegen der langen Wege kommt nur ein geringer Bruchteil $\left(10^{-10}\right)$ der ausgesandten L eistung an; daher reicht die Lichtintensität für einen rein interferometrischen $\mathrm{N}$ achweis nicht aus. Statt dessen vergleicht man die Phasenverschiebung zwischen den einzelnen L asern, die aufeinander abgestimmt sind. D ie Satellitengehäuse schirmen die Interferometerspiegel vor dem Strahlungsdruck der Sonne ab. Die Testmassen bewegen sich auf $\mathrm{G}$ eodäten im R aum-Zeit-K ontinuum und stellen somit ideale Inertialsysteme dar. E in ausgeklügeltes K ontrollsystem zwingt die Satelliten, dieser Bewegung zu folgen. D as Projekt stellt eine faszinierende $M$ ischung aus optischer Technologie, Raumfahrt- und Regelungstechnik dar.

$M$ it der Kombination von mehreren A ntennen auf der Erde und dem LISA-Projekt im Weltraum besteht also die günstige A ussicht, in den nächsten 15 Jahren das neue Fenster ins All in seiner vollen Breite zu öffnen und Informationen über das U niversum zu erhalten, die uns bisher nicht zugänglich waren.

\section{Literatur}

Einen guten Überblick über den Stand der Gravitationswellenforschung zu A nfang der 80er Jahre geben die A rtikel:

W. Winkler, Physik in unserer Zeit 16, 138 (5/1985).

D. G. Blair, Physik in unserer Zeit 17, 142 (5/1986).

Ü ber den aktuellen Stand berichten:

K. D anzmann, H. Ruder, Phys. BI. 49, 103 (1993).

F. Ricci u. A. Brillet, Ann. Rev. N ucl. Part. Sci. 47, 111 (1997).

\section{Weitere Literatur}

[1] J. A. Wheeler, Einsteins Vision. Springer-Verlag, Berlin (1968).

[2] P. Kafka, N aturwissenschaften 73, 248 u. 305 (1986).

[3] B. F. Schutz, Class. Q uant. Grav. 13, A 219 (1996).

[4] J. Weber, Phys. Rev. 117, 307 (1960).

[5] L. Ju u. D.G. Blair, Int. Journ. M od. Phys. D 5, 101 (1996)

[6] M.H. Soffel U. H. Ruder, Physik in unserer Zeit 22, 29 (1/1991) u. 24, 253 (6/1993).

[7] B.F. Schutz, N ature 323, 310 (1986).

[8] G. Heinzel et al. Phys. Rev. Lett. 81, 5493 (1998).

[9] T. Zwerger u. E. M üller, A stron. A strophys. 320, 209 (1997).
A lle Projekte sind auch im Internet beschrieben:

GEO 600: http://www.ge0600.unihannover.de

LIG O : http://www.ligo.caltech.edu LISA : http://lisa.jpl.nasa.gov TA M A 300: http://tamago.mtk.nao.ac.jp VIRG 0 : http://www.virgo.infn.it

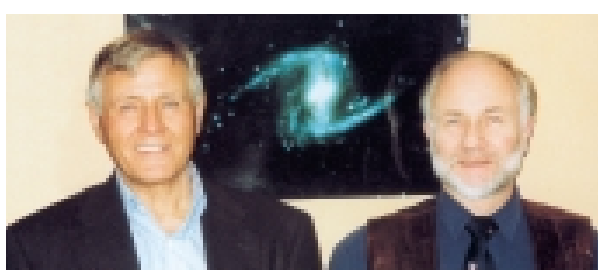

Peter A ufmuth (rechts), geb. 1943, Studium der Physik an der Universität $\mathrm{H}$ annover, Promotion 1977; wissenschaftlicher M itarbeiter am I nstitut für A tom- und Molekülphysik der $\mathrm{U}$ niversität $\mathrm{H}$ annover; zunächst Experimente und Rechnungen zur I sotopieverschiebung und $\mathrm{H}$ yperfeinstruktur freier A tome, seit 1991 M itarbeit am G EO -Projekt.

A lbrecht Rüdiger, geb. 1929, Studium der Physik an der U niversität Frankfurt am M ain; seit 1957 an den Max-Planck-Instituten für Physik, für A strophysik, für Q uantenoptik; Grundlagenforschungen über Computer-Elemente, über vollautomatische A uswertung von Blasenkammerbildern, seit 1977 über G ravitationswellendetektoren.

Anschriften:

Dr. Peter A ufmuth, Institut für A tom- und M olekülphysik, A bt. Spektroskopie, C allinstraße 38, D-30167 H annover; pea@mpq.mpg.de.

A lbrecht Rüdiger, M ax-Planck-I nstitut für Q uantenoptik, $\mathrm{H}$ ans-K opfermann-Straße $1, \quad \mathrm{D}-85748$ G arching; atr@mpq.mpg.de. 